\title{
Improvement Control System of Rabies in Ukraine
}

\author{
Serhiy Nychyk*1, Ostap Zhukorskiy², Ivan Polupan ${ }^{1}$, Mykola Ivanov $^{1}$ and Alina Nikitova ${ }^{1}$ \\ ${ }^{1}$ Institute of Veterinary Medicine, Kyiv, Ukraine; ${ }^{2}$ National Academy of Agrarian Sciences of Ukraine, Kyiv, Ukraine
}

\section{Objective}

The purpose of the research was to find out the reasons of rabies antropurgisation in Ukraine.

\section{Introduction}

In Ukraine in spite of considerable financial expenses on oral immunization of foxes and parenteral immunization of dogs and cats, it is not succeeded to reach considerable results in the fight with rabies. Unfortunately there was a negative tendency to increasing a part of dogs and cats in the structure of rabies disease which are the main source of rabies in people.

\section{Methods}

Analysis of 228 anamnesis data of rabies infected dogs during 2008-2012. Research of 234 samples of the blood serum from dogs on existence of antibodies to the rabies virus by the ELISA method.

\section{Results}

Analysis of animal morbidity on rabies in Ukraine in period of 2006-2011 found out the changes of structure of morbidity in animal species that means decreasing a part of wild animals (from $49,0 \%$ in 2006 to $38,7 \%$ in 2011) and increasing a part of dogs (from $18,3 \%$ in 2006 to $23,2 \%$ in 2011) and cats (from $19,8 \%$ in 2006 to $25,0 \%$ in 2011) in the general amount of animals which perished from rabies (Fig. 1).

A lot of Ukrainian scientists and doctors of veterinary medicine consider that the main reason of spreading the rabies is a great number of homeless animals which factually are the reservoirs of infection in towns and villages.

However, in our opinion spreading of rabies shows the insufficient level of measures of control of rabies among home animals. It was confirmed with conducted analysis that only $26(12,9 \%)$ dogs were stray, others $202(87,1 \%)$ had owners, but didn't get necessary protective rabies vaccination.

According to Ukrainian instruction "Preventive measures against rabies of animals", all the dogs must be vaccinated against rabies, but it actually appears it is quite not so.

At research of 234 samples of the blood serum of dogs on existence of antibodies to the rabies virus it was determined that the level of population immunity in dogs is $36,6 \%$ in Ukrainian towns, but the protective level of antibodies was found in $9,1 \%$ of village dogs. We consider that the main reason of this is imperfection of the Ukrainian legislation in the questions of responsibility of proprietors of animals.

For breaking of epizootic chain it is necessary the percent of vaccinated animals (in this case dogs) to be on a high level - more than $50 \%$. Nowadays for reaching epidemic welfare in rabies it is important within the framework of the registered epidemic incidents dogs to be brought over to the ecocycles of infection sporadically without taking part in circulation of exciter, stay their biological deadlock and have low epidemic potential.

\section{Conclusions}

The conducted analysis expressly demonstrates that at present problems the eradication of rabies in Ukraine is impossible considering the low level of dogs' protection from rabies. Obtained results were sent to the State committee of veterinary medicine of Ukraine and will be the argument for enhancement of control after conducting rabies vaccination of dogs.

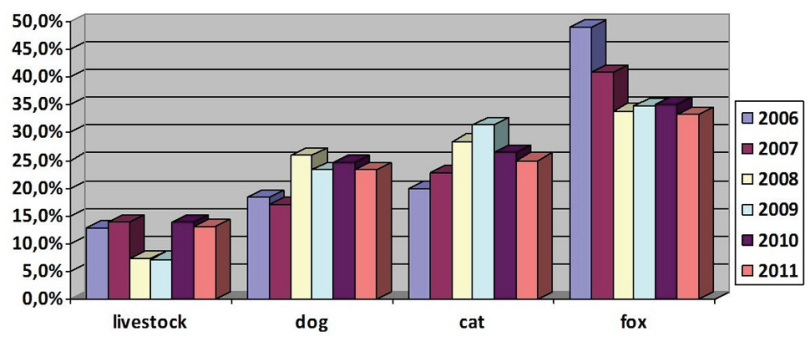

Rabies cases in the years 2006-2011.

\section{Keywords}

population rabies immunity; rabies vaccination; stray dogs

*Serhiy Nychyk

E-mail: vetmedic@ukr.net 